ISSN 0103-9954

\title{
AVALIAÇÃO DA PRODUTIVIDADE, CONTEÚDO E EFICIÊNCIA DE UTILIZAÇÃO DE NUTRIENTES EM GENÓTIPOS DE Eucalyptus spp. NO VALE DO JEQUITINHONHA, MG
}

\author{
EVALUATION OF THE PRODUCTIVITY, CONTENT AND NUTRIENT USE EFFICIENCY IN \\ GENOTYPES OF Eucalyptus spp. IN JEQUITINHONHA VALLEY, MG
}

\author{
Geraldo Erli de Faria ${ }^{1}$ Nairam Félix de Barros ${ }^{2}$ Vanderley Luiz Paranaíba Cunha ${ }^{3}$ \\ Ildeu Soares Martins ${ }^{4}$ Rosana de Carvalho Cristo Martins ${ }^{5}$
}

\begin{abstract}
RESUMO
A hibridação de espécies de eucalipto possibilita a obtenção de híbridos mais produtivos e com alta eficiência de absorção e/ou utilização de nutrientes, dependendo das espécies combinadas. Neste contexto, o presente trabalho, conduzido na região do Vale do Jequitinhonha, Minas Gerais, teve por objetivos: determinar a produtividade, o conteúdo e a eficiência de utilização de $\mathrm{N}, \mathrm{P}, \mathrm{K}, \mathrm{Ca}$ e $\mathrm{Mg}$ de híbridos interespecíficos de Eucalyptus spp. Para tanto, mediu-se o diâmetro à altura do peito (DAP) de todas as árvores presentes em cada parcela, e abateram-se três árvores com diâmetro correspondente ao da árvore média. Procedeu-se à cubagem das árvores pelo método de Smallian, utilizando a altura, o diâmetro e a espessura de casca na base e a 25, 50, 75 e $100 \%$ da altura comercial. O peso de material fresco do lenho, da casca, das folhas e dos galhos foi determinado no campo, e desses componentes se coletaram amostras para determinação do peso da matéria seca. As amostras do tronco consistiram de discos coletados, com casca, na base e a 25, 50, 75 e $100 \%$ da altura comercial. As amostras de tecido vegetal foram analisadas para: N, P, K, Ca e Mg. Os resultados obtidos não indicaram diferenças significativas para a produção de biomassa de copa entre os híbridos de eucalipto em conseqüência de apresentarem mesma idade. Entretanto, a produção de biomassa de tronco apresentou diferenças significativas sendo os híbridos de Eucalyptus urophylla S. T. Blake proveniente de polinização natural mais produtivo em 50 e 69\% em comparação aos híbridos de Eucalyptus urophylla W. Hill. Ex Maiden com Eucalyptus grandis Dehnh.e de Eucalyptus urophylla com descendentes do cruzamento de Eucalyptus camaldulensis com Eucalyptus grandis respectivamente. Além disso, aqueles híbridos foram os que alocaram maior quantidade de biomassa no tronco $(92,3 \%)$ em relação à copa $(7,7 \%)$, e ainda foram os mais eficientes na absorção e utilização de N, P, K, Ca e Mg. Concluiu-se que: 1) híbridos de Eucalyptus urophylla provenientes de polinização natural ou controlada não apresentam diferença na produção de biomassa de copa quando avaliados numa mesma idade; 2) a polinização controlada de Eucalyptus urophylla com Eucalyptus grandis e com descendentes do cruzamento de Eucalyptus camaldulensis com Eucalyptus grandis reduz a produção de biomassa de tronco; 3) híbridos de Eucalyptus urophylla provenientes de polinização natural são mais produtivos e mais eficientes na absorção e utilização de N, P, K, Ca e Mg e 4) híbridos de Eucalyptus urophylla provenientes de descendentes do cruzamento de Eucalyptus camaldulensis com Eucalyptus grandis são menos produtivos e menos eficientes na absorção e utilização de N, P, K, Ca e Mg.
\end{abstract}

Palavras-chave: eucalipto; híbridos; eficiência nutricional.

\section{ABSTRACT}

The hybridization of eucalyptus species can provide more productive hybrids and with a high efficiency of nutrient absorption and, or, nutrient use, depending on the combined species. In this context, the present work was undertaken in the Jequitinhonha Valley region, Minas Gerais, and its objectives were: to determine the productivity, content and use efficiency of N, P, K, Ca and Mg in interspecific hybrids of Eucalyptus spp. In each plot, the diameters were measured at breast height (DBH) in all the trees, and three trees with diameter similar to the average mean were felled. The volume of the trees was estimated by the

1. Engenheiro Florestal, Dr., Professor Adjunto, Universidade Federal do Maranhão, Campus IV, MA-230, km 04, S/N, Boa Vista, CEP 65500-000, Chapadinha (MA).geraldoerli@yahoo.com.br

2. Engenheiro Florestal, PhD., Professor do Departamento de Solos, Universidade Federal de Viçosa, Av. Peter Henry Rolfs s/n, Campus Universitário, CEP 36570 000, Viçosa (MG).nfbarros@ufv.br

3. Engenheiro Florestal, Gerente de Desenvolvimento da Empresa Acesita Energética Ltda., Rua Raul Coelho, 725, Bairro Centro, CEP 39680-000, Capelinha (MG). wanderleycunha@acesita.com.br

4. Engenheiro Florestal, Dr., Professor Adjunto do Departamento de Engenharia Florestal, Universidade de Brasília, Faculdade de Tecnologia, Caixa Postal, 04357, CEP 70919-970, Brasília (DF).

5. Engenheira Florestal, Dr ${ }^{\mathrm{a}}$., Professora Adjunta do Departamento de Engenharia Florestal, Faculdade de Tecnologia, Caixa Postal, 04357, Universidade de Brasília, CEP 70919-970, Brasília (DF). rccristo@unb.br

Recebido para publicação em 7/11/2006 e aceito em 11/08/2008. 
Smallian method, using the height, diameter and the bark thickness at the base and at 25, 50, 75 and $100 \%$ of the commercial height. The fresh weight of the wood, bark, leaves and branches was determined in the field, and samples from them were collected for the determination of dry matter. The log samples consisted of collected disks with bark, from the base and at 25, 50, 75 and $100 \%$ of the commercial height. The samples of vegetable tissues were analyzed for: N, P, K, Ca and Mg. The results obtained did not show significant differences for the production of crown biomass between the eucalyptus hybrids of the same age. However, the production of log biomass presented significant differences and the naturally pollinated hybrids of Eucalyptus urophylla were $50.0 \%$ and $69.0 \%$ more productive compared with the hybrids of Eucalyptus urophylla with Eucalyptus grandis and Eucalyptus urophylla with descendants of the crossing of Eucalyptus camaldulensis with Eucalyptus grandis, respectively. Also, these hybrids allocated larger amount of biomass in the $\log (92.3 \%)$ in relation to the crown (7.7\%), and they were more efficient in the absorption and use of N, P, K, Ca and Mg. The conclusions were that: 1) same aged hybrids of Eucalyptus urophylla from natural or controlled pollinations did not differ in their crown biomass; 2) log biomass was reduced in the hybrids from controlled pollination between Eucalyptus urophylla with Eucalyptus grandis and Eucalyptus urophylla with descendants of the crossing of Eucalyptus camaldulensis with Eucalyptus grandis; 3) hybrids of Eucalyptus urophylla from natural pollination were more productive and more efficient in the absorption and efficiency of use of N, P, K, Ca and Mg and 4) hybrids of Eucalyptus urophylla with descendants of the crossing of Eucalyptus camaldulensis with Eucalyptus grandis are less productive and less efficient in the absorption and use of N, P, K, Ca and Mg.

Keywords: Eucalyptus; hybrids; efficiency nutritional.

\section{INTRODUÇÃO}

A região do cerrado apresenta como características principais baixa precipitação pluviométrica, elevada evapotranspiração potencial, períodos com déficit hídrico acentuado e solos altamente intemperizados, com baixa fertilidade natural, conseqüência de sua pobreza em nutrientes minerais. Essas condições adversas de clima e solo, quando combinadas, constituem-se em forte limitação à produtividade florestal, a menos que sejam adotadas práticas silviculturais como a fertilização mineral e a seleção de materiais genéticos mais adaptados e eficientes no uso de nutrientes sob tais condições, sendo as espécies desejáveis aquelas com maior capacidade de absorver e utilizar os nutrientes (MORAIS et al., 1990).

De acordo com Hansen e Baker (1979), a eficiência de utilização dos nutrientes em uma determinada espécie vegetal pode ser definida como a quantidade de matéria seca em quilograma produzida por quilograma de nutriente utilizado. Por meio desse parâmetro, pode-se analisar quantos quilogramas de nutriente foram necessários para produzir a matéria seca de uma determinada espécie vegetal (PEREIRA et al., 1984).

A avaliação da eficiência de utilização dos nutrientes por parte das diferentes espécies florestais, procedências e/ou clones é um importante parâmetro para auxiliar o silvicultor no momento de optar pelo material a ser utilizado nos reflorestamentos (CALDEIRA et al., 2004).

A constatação de diferenças no comportamento nutricional entre híbridos de eucalipto (LIMA et al., 2005) tem grande relevância prática, pois permite a alocação desses materiais em solos de baixa fertilidade natural e a adoção de regimes diferenciados de adubação. Além disso, os indivíduos altamente eficientes na utilização de nutrientes podem ser produtivos, com menor requerimento de nutrientes, ou apresentar maior conversão dos nutrientes em biomassa, em virtude do rápido transporte no xilema e rápida assimilação e/ou grande capacidade de ciclagem interna (GRAHAM, 1984). Essa habilidade seria conseqüência direta da evolução de genótipos em ambientes distintos, nos quais vários fatores do meio como: temperatura, água e disponibilidade de nutrientes levariam à diferenciação no comportamento desses indivíduos (MASON e PELHAM, 1976) e, ainda, poderia proporcionar aumento na capacidade produtiva. Contudo, no caso de não haver a correspondência entre a alta eficiência de absorção e a alta produtividade, maior exportação de nutrientes estaria ocorrendo proporcionalmente por unidade de biomassa produzida, caracterizada por um possível consumo de luxo, ou seja, maior absorção de nutrientes sem um conseqüente aumento na biomassa produzida (SANTANA et al., 2002).

A avaliação da eficiência de absorção e utilização de nutrientes por híbridos interespecíficos de eucalipto proporcionalmente à biomassa produzida é desejável para a manutenção da sustentabilidade da 
produção florestal, sobretudo, em condições de escassez de nutrientes no solo e limitações hídricas, como as ocorrentes no bioma Cerrado.

O objetivo deste trabalho foi determinar a produtividade, o conteúdo e a eficiência de utilização de N, P, K, Ca e Mg de híbridos interespecíficos de Eucalyptus spp. no Vale do Jequitinhonha, Minas Gerais.

\section{MATERIAL E MÉTODOS}

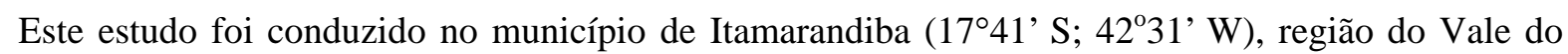
Jequitinhonha, Minas Gerais, com altitude de 910 m. A região, segundo a classificação de Köppen, apresenta déficit hídrico anual de 60 a $120 \mathrm{~mm}$, ocorrendo de 4 a 6 meses no inverno e temperatura média de 19 a $22^{\circ} \mathrm{C}$, com estação seca bem definida. O solo foi classificado como Latossolo Vermelho-Amarelo Distrófico (EMBRAPA, 1999), textura argilosa, e, a área de estudo estava sob o domínio do ecossistema de cerrado (ANTUNES, 1980).

O estudo contemplou híbridos interespecíficos do gênero Eucalyptus spp. obtidos pela polinização de Eucalyptus urophylla (mãe) com pólen coletado de árvores de híbridos naturais (pai), ou com pólen de árvores selecionadas de Eucalyptus grandis (pai) e de híbrido de Eucalyptus grandis e Eucalyptus camaldulensis (pai). No primeiro cruzamento, utilizou-se o método polimix (ASSIS, 1993), no qual uma mistura de pólen de sete progenitores foi utilizada no processo (Tabela 1).

Os materiais genéticos foram plantados no espaçamento de $3 \times 2 \mathrm{~m}$ em blocos casualizados em parcelas com dimensão de 2,25 ha. No preparo do solo, foram aplicadas $2 \mathrm{t} \mathrm{ha}^{-1}$ de calcário, a lanço, seguido de incorporação com grade leve aos $20 \mathrm{~cm}$ superficiais. No plantio das mudas, foram aplicados $200 \mathrm{~kg} \mathrm{ha}^{-1}$ da formulação NPK 4-26-16 na linha de plantio e, decorridos um ano, fez-se a adubação complementar de $150 \mathrm{~kg} \mathrm{ha}^{-1}$ da formulação NPK 10-5-30 em todas as parcelas.

Em idade em torno de 57 meses, dentro de cada parcela, estabeleceram-se três subparcelas de $400 \mathrm{~m}^{2}$ nas quais se mediu o diâmetro à altura do peito (DAP) de todas as árvores e abateram-se três com o diâmetro correspondente ao da árvore média da subparcela para a determinação da biomassa de seus componentes e amostragem de material vegetal para análise química. Para tanto, procedeu-se à cubagem das árvores pelo método de Smallian, utilizando a altura, o diâmetro e a espessura de casca na base e a 25, 50, 75 e 100\% da altura comercial. O peso de material fresco do lenho, casca, folhas e galhos foram determinados no campo, e, desses componentes, coletaram-se amostras para determinação do peso da matéria seca e para análise química. As amostras do tronco consistiram de discos coletados, com casca, na base e a 25, 50, 75 e 100\% da altura comercial. Para a análise química, coletou-se uma amostra de "pó-de-serra" obtida por meio de corte com motosserra até o meio da seção transversal, de 50 em $50 \mathrm{~cm}$, ao longo do tronco.

TABELA 1: Relação dos híbridos interespecíficos de Eucalyptus spp. submetidos ao estudo em diferentes categorias e combinações.

TABLE 1: Relationship of the interspecific hybrids of Eucalyptus spp. submitted to the study in different categories and combinations.

\begin{tabular}{|c|c|c|c|}
\hline Clone & Categoria & Materiais genéticos & Combinação Mãe x Pai \\
\hline I-224 & P. Natural & UR x --- & - \\
\hline$I-060$ & P. Natural & UR x --- & - \\
\hline I-144 & P. Natural & UR x --- & - \\
\hline I-225 & P. Natural & UR x --- & - \\
\hline I-040 & P. Natural & UR x --- & - \\
\hline HC-249 & P. controlada & UR x GR & $31 \times 45$ \\
\hline HC-232 & P. controlada & UR x GR & $13 \times 13$ \\
\hline HC-373 & P. controlada & UR x GR & $41 \times 45$ \\
\hline HC-257 & P. controlada & UR x GR & $31 \times 45$ \\
\hline HC-344 & P. controlada & UR x GR & $13 \times 19$ \\
\hline HC-289 & P. controlada & UR x GR & $36 \times 45$ \\
\hline НCT-041 & P. cont. tricross & UR $x\left(\mathrm{CA}^{4 /} \times \mathrm{GR}\right)$ & $06 \times \mathrm{I}-760$ \\
\hline НCT-027 & P. cont. tricross & UR x (CA x GR) & $06 \times \mathrm{I}-760$ \\
\hline HCT-037 & P. cont. tricross & UR x (CA x GR) & $06 \times \mathrm{I}-760$ \\
\hline
\end{tabular}

Em que: $\mathrm{P}$. = polinização; UR = Eucalyptus urophylla; $\mathrm{GR}$ = Eucalyptus grandis; $\mathrm{CA}$ = Eucalyptus camaldulensis. 
As amostras dos componentes das árvores foram acondicionadas em saco de papel e secadas em estufa de circulação forçada a $70^{\circ} \mathrm{C}$ até peso constante. $\mathrm{O}$ material seco foi pesado, moído e submetido à digestão nitroperclórica. Nos extratos, foram determinados os teores de $\mathrm{K}$ por fotometria de emissão de chama (TEDESCO et al., 1995), o de P por colorimetria (BRAGA e DEFELIPO, 1974), e o de Ca e Mg por espectrofotometria de absorção atômica (A.O.A.C, 1975). Os teores de $\mathrm{N}$ foram determinados pelo método de Kjeldhal.

Estimou-se o conteúdo de cada nutriente, multiplicando-se o seu teor pelo peso de matéria seca dos componentes da árvore, e a eficiência de utilização dos nutrientes foi realizada com base na estimativa do coeficiente de utilização biológico (CUB), empregado por Barros et al. (1986), sendo CUB = matéria seca de tronco $\left(\mathrm{kg} \mathrm{ha}^{-1}\right) /$ conteúdo do nutriente no tronco $\left(\mathrm{kg} \mathrm{ha}^{-1}\right)$.

Os dados de biomassa de tronco, parte aérea, conteúdo de nutrientes e o coeficiente de utilização biológica (CUB) de $\mathrm{N}, \mathrm{P}, \mathrm{K}, \mathrm{Ca}$ e $\mathrm{Mg}$ foram submetidos à análise de variância e as médias foram discriminadas por meio do teste Scott-Knott, a 5\% de probabilidade.

\section{RESULTADOS E DISCUSSÃO}

\section{Biomassa de copa, lenho e tronco - híbridos de Eucalyptus spp.}

Quanto à produção de biomassa de copa, não se verificou diferença significativa entre os híbridos avaliados (Tabela 2). Fato que pode ser explicado pela semelhança de idade na época de avaliação do experimento (57 meses).

TABELA 2: Incremento médio anual (IMA), biomassa de copa, lenho e tronco e partição relativa dos componentes copa e tronco de híbridos interespecíficos de Eucalyptus spp. aos 57 meses.

TABLE 2: Annual average increment, crown, wood and log biomass and relative partition of the crown and log components of Eucalyptus spp. hybrids at 57 months.

\begin{tabular}{|c|c|c|c|c|c|c|c|}
\hline \multirow{3}{*}{ Híbrido } & \multirow{3}{*}{ Clone } & \multirow{2}{*}{ IMA } & \multicolumn{3}{|c|}{ Biomassa } & \multicolumn{2}{|c|}{ Partição } \\
\hline & & & Copa & Lenho & Tronco & Copa & Tronco \\
\hline & & $\mathrm{m}^{3} \mathrm{ha}^{-1} \mathrm{ano}^{-1}$ & \multicolumn{3}{|c|}{$\mathrm{tha}^{-1}$} & \multicolumn{2}{|c|}{$\%$} \\
\hline UR x --- & $\mathrm{I}-224$ & $72,8 \mathrm{~A}$ & $13,8 \mathrm{~A}$ & $157,1 \mathrm{~A}$ & $174,0 \mathrm{~A}$ & 7,4 & 92,7 \\
\hline UR x --- & $\mathrm{I}-060$ & 64,4 B & $9,1 \mathrm{~A}$ & 134,4 B & 149,1 B & 5,6 & 94,3 \\
\hline UR x --- & I-144 & 64,3 B & $16,2 \mathrm{~A}$ & 144,8 A & $160,1 \mathrm{~A}$ & 9,2 & 90,8 \\
\hline UR x --- & I-225 & $55,1 \mathrm{C}$ & $11,5 \mathrm{~A}$ & 128,4 B & 140,4 B & 7,6 & 92,4 \\
\hline UR x --- & I-044 & $52,6 \mathrm{C}$ & $12,4 \mathrm{~A}$ & 115,8 B & $127,8 \mathrm{~B}$ & 8,8 & 91,2 \\
\hline Média & & 61,8 & 12,6 & 136,1 & 150,3 & 7,7 & 92,3 \\
\hline UR x GR & HC-249 & $47,9 \mathrm{D}$ & $14,5 \mathrm{~A}$ & $97,7 \mathrm{C}$ & $109,6 \mathrm{C}$ & 11,7 & 88,3 \\
\hline UR x GR & HC-232 & $46,2 \mathrm{D}$ & $12,0 \mathrm{~A}$ & $84,1 \mathrm{D}$ & $96,8 \mathrm{D}$ & 11,0 & 89,0 \\
\hline UR x GR & HC-373 & $46,1 \mathrm{D}$ & 14,2 A & 97,6 C & 108,7 C & 11,6 & 88,5 \\
\hline UR x GR & HC-257 & $44,5 \mathrm{E}$ & $16,1 \mathrm{~A}$ & 87,6 D & $98,4 \mathrm{D}$ & 14,1 & 85,9 \\
\hline UR x GR & HC-344 & $42,9 \mathrm{E}$ & $13,2 \mathrm{~A}$ & 79,7 D & $90,9 \mathrm{D}$ & 12,7 & 87,3 \\
\hline UR x GR & HC-289 & $41,7 \mathrm{E}$ & $12,9 \mathrm{~A}$ & $83,2 \mathrm{D}$ & $95,7 \mathrm{D}$ & 11,9 & 88,1 \\
\hline Média & & 45,7 & 13,7 & 90,5 & 102,5 & 11,8 & 88,2 \\
\hline UR x (CA x GR) & HCT-041 & $46,5 \mathrm{D}$ & $10,4 \mathrm{~A}$ & $93,5 \mathrm{C}$ & $105,3 \mathrm{C}$ & 9,0 & 91,0 \\
\hline UR x (CA x GR) & НCT-027 & $37,0 \mathrm{~F}$ & $8,3 \mathrm{~A}$ & $71,4 \mathrm{D}$ & $82,0 \mathrm{D}$ & 9,2 & 90,8 \\
\hline UR x (CA x GR) & HCT-037 & $36,4 \mathrm{~F}$ & $9,2 \mathrm{~A}$ & $69,4 \mathrm{D}$ & $79,5 \mathrm{D}$ & 10,4 & 89,6 \\
\hline Média & & 40,0 & 9,3 & 78,1 & 88,9 & 9,5 & 90,5 \\
\hline
\end{tabular}

Em que: Médias seguidas das mesmas letras, em cada coluna, não diferem entre si, pelo teste Scott-Knott a $5 \%$ de probabilidade. I = híbridos de Eucalyptus urophylla em polinização natural; HC = híbridos de Eucalyptus urophylla com Eucalyptus grandis; HCT = híbridos de Eucalyptus urophylla com descendentes de Eucalyptus camaldulensis com Eucalyptus grandis.

Na distribuição relativa da biomassa de copa e do tronco, verificou-se que os híbridos de Eucalyptus urophylla provenientes de polinização de híbridos naturais, além de serem os mais produtivos, tenderam alocar maior porcentagem, em termos médios, de biomassa de tronco (92,3\%) em relação à copa $(7,7 \%)$ (Tabela 2). 
A alocação de biomassa no tronco (92,3\%) dos híbridos de Eucalyptus urophylla provenientes de polinização de híbridos naturais é 2,6\% maior que àquelas verificadas em Eucalyptus camaldulensis, Eucalyptus grandis e Eucalyptus torelliana (90\%) (SCHUMACHER e POGGIANI, 1993) e 5,1\% em relação a Eucalyptus grandis, Eucalyptus camaldulensis e Eucalyptus pellita (87,9\%) (ZAIA e GAMA-RODRIGUES, 2004), mostrando que a hibridação possibilita o melhoramento de características florestais desejáveis (ASSIS, 2000), mesmo em condições adversas como as ocorrentes na região do cerrado.

Segundo Ladeira (1999), as diferenças de partição de biomassa para o tronco são muito importantes para a tomada de decisões quanto à seleção de materiais genéticos e técnicas de manejo a serem adotadas na condução do povoamento, uma vez que o tronco, de modo geral, é o componente da árvore explorado comercialmente.

A produtividade dos híbridos interespecíficos de eucalipto variou significativamente entre os genótipos avaliados (Tabela 2). Comparando-se os clones, em termos de incremento médio anual (IMA), constatou-se redução de 34,2 e 36,1\% partindo do clone mais produtivo (I-224) para o clone HC-249 e HCT041 respectivamente, os quais apresentaram maior IMA entre os clones provenientes do cruzamento de Eucalyptus urophylla com Eucalyptus grandis (HC) e Eucalyptus urophylla com descendentes de Eucalyptus camaldulensis com Eucalyptus grandis (HCT). Além disso, houve redução de 27,7\% do clone I-224 em relação ao clone I-044, os quais são provenientes de híbridos de Eucalyptus urophylla em polinização natural. Por outro lado, quando se comparou o clone mais produtivo (I-224) com o menos produtivo (HCT037), verificou-se redução de $50,0 \%$ e $54,3 \%$ para o IMA e para a produção de biomassa de tronco, respectivamente (Tabela 2). Essas variações indicam que a hibridação interespecífica é altamente importante para a elevação da produção de biomassa, dependendo das espécies combinadas (MOLICA, 1992).

Em termos médios, os híbridos de Eucalyptus urophylla provenientes de polinização de híbridos naturais foram os que apresentaram maior produção de biomassa de tronco (150,3 t ha $\left.{ }^{-1}\right)$. Entretanto, quando houve a polinização do Eucalyptus urophylla (mãe) com o pólen proveniente de Eucalyptus grandis (pai) e de descendentes do cruzamento de Eucalyptus camaldulensis com Eucalyptus grandis (pai), houve redução de 31,8 e 40,9\% nesse componente respectivamente (Tabela 2). As diferenças verificadas podem ser atribuídas as espécies e/ou procedências de eucalipto utilizadas na polinização controlada não serem as mais indicadas para serem cultivadas na região do Vale do Jequitinhonha que apresenta solos com baixo teores de nutrientes em formas disponíveis e totais (BARROS et al., 1990; MOLICA, 1992) e períodos com déficit hídrico acentuado (GOLFARI, 1975). Nesse caso, o mais indicado é a obtenção de híbridos interespecíficos de eucalipto pelo método polimix, o qual consiste na polinização de árvores matrizes da espécie materna (Eucalyptus urophylla) com pólen proveniente de uma mistura de sete árvores-matrizes da espécie paterna (híbridos naturais) de procedências distintas, os quais alcançaram maiores produtividades na mesma idade (57 meses) que os demais híbridos avaliados.

Oda et al. (1989) afirmam que a produtividade em plantas, em geral, está relacionada diretamente com maior grau de heterozigose cuja expressão máxima ocorre em casos de hibridação interespecífica. Sendo que a heterose ou vigor híbrido pode se manifestar sobretudo em determinados ambientes com condições de estresse nutricional ou climático. Fato que pode ter contribuído para as maiores produtividades obtidas para os híbridos interespecíficos de eucalipto provenientes de polinização natural na região do Vale do Jequitinhonha, Minas Gerais, conforme constatado por Assis (1996) e Molica (1992).

\section{Conteúdo de nutrientes (parte aérea e no tronco) e eficiência nutricional - híbridos de Eucalyptus spp.}

Os materiais genéticos diferiram consideravelmente quanto ao acúmulo de nutrientes na parte aérea e no tronco e, em termos médios, apresentaram a seguinte seqüência: $\mathrm{N}>\mathrm{Ca}>\mathrm{K}>\mathrm{Mg}>\mathrm{P}$ (Tabela 3). Resultados semelhantes foram obtidos em outros estudos (REIS e BARROS, 1990; SANTANA et al., 2002). Schumacher e Poggiani (1993) comentam que o acúmulo de nutrientes na biomassa arbórea varia de elemento para elemento em função das características nutricionais de cada espécie, dos diferentes níveis de fertilidade do solo e da idade da floresta. Além disso, tem-se que o conteúdo de nutrientes é conseqüência da concentração e da produção de biomassa (MOURA, 2006).

O P, por ter maior mobilidade na planta do que o Ca, se apresenta normalmente em percentagens e quantidades maiores em outros componentes da planta, como folhas e galhos (PEREIRA et al., 1984), os quais, em geral, não são comercializáveis e deixados no próprio local de colheita. Por exemplo, o Ca e o K 
são os nutrientes presentes em maior quantidade no tronco, seguidos pelo Mg e P. É também o Ca o nutriente mais abundante na casca, enquanto no lenho é o K, sendo os elementos mais exportados (SANTANA et al., 2002).

TABELA 3: Conteúdo de nutrientes na biomassa aérea total e no tronco de híbridos interespecíficos de Eucalyptus spp. aos 57 meses de cultivo.

TABLE 3: Nutrient content in the total aerial biomass and in the log of interspecific hybrids of Eucalyptus spp. at 57 months of cultivation.

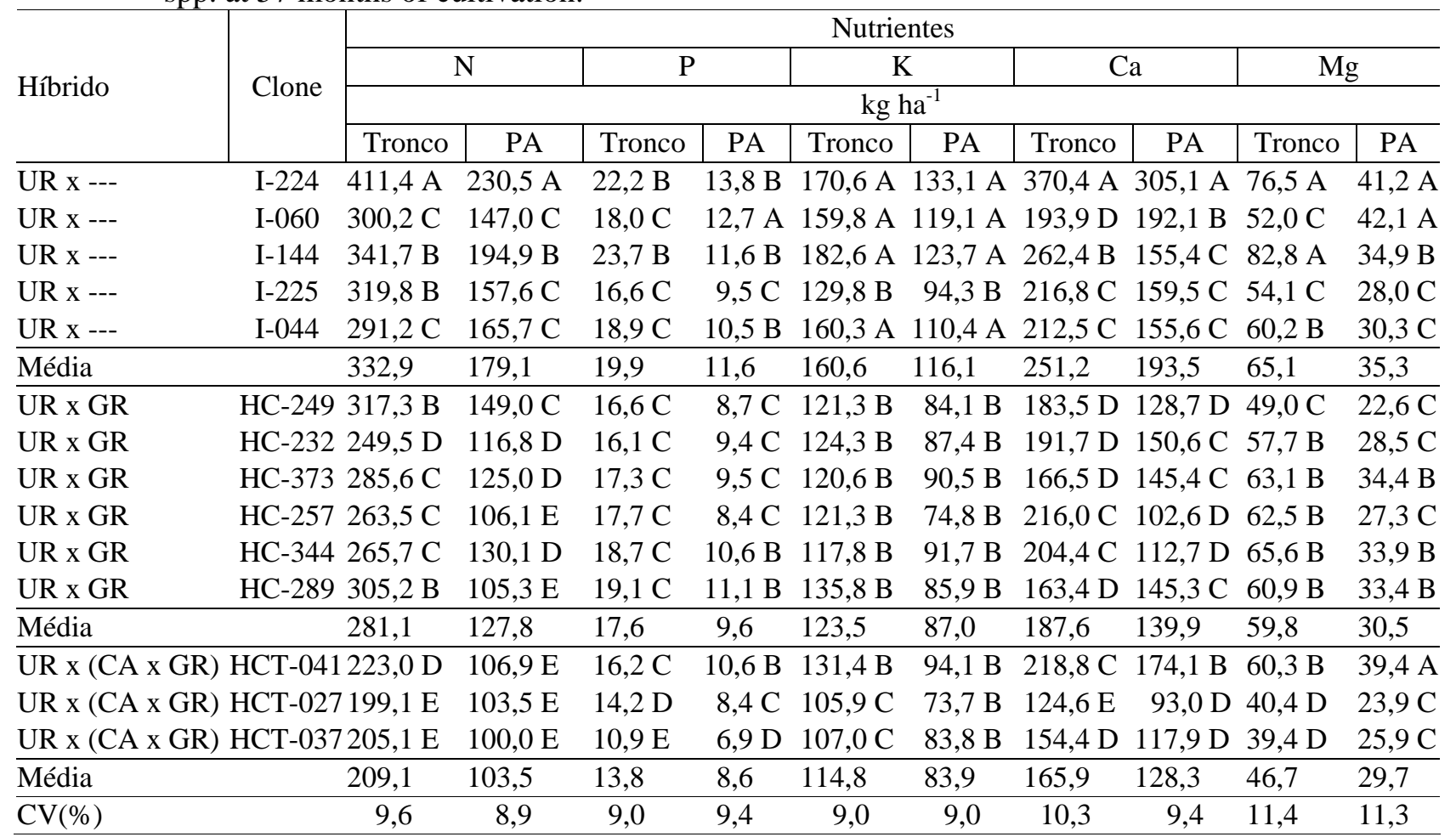

Em que: $\mathrm{PA}$ = conteúdo de nutrientes na biomassa aérea total. Médias seguidas das mesmas letras, em cada coluna, não diferem entre si, por meio do teste Scott-Knott a 5\%.

Uma forma de reduzir a exportação de nutrientes do sítio e, assim, manter a qualidade e a capacidade produtiva do solo durante um maior período de tempo é promover a exploração somente da parte comercializável da planta. Nesse sentido, o descascamento do tronco e a manutenção da casca no sítio é uma prática recomendada visando a reduzir a exportação de nutrientes sobretudo de Ca que, em virtude da sua nãoremobilização interna pelas plantas de eucalipto, tende a concentrar-se em maior quantidade na casca (PEREIRA et al., 1984; REIS e BARROS, 1990; SANTANA et al., 2002). O descascamento do tronco e a manutenção da casca no sítio podem reduzir em até 86,5\% a remoção de Ca do sistema e em 23,3, 49,5 e 67,8\% a exportação de P, K e Mg respectivamente. Além disso, Silveira e Malavolta (2000) apontam que, em média, cerca de $24 \%$ do conteúdo total de K se encontram nas folhas e $16 \%$ nos ramos do eucalipto. Fato que se reveste de importância quando se considera a região do Vale do Jequitinhonha (Itamarandiba-MG), em conseqüência desta apresentar baixa disponibilidade de nutrientes, sobretudo K (BARROS et al., 1990; MOLICA, 1992; GALO, 1993) e déficit hídrico acentuado (GOLFARI, 1975), sendo, nessas condições, importante a manutenção de maiores teores de K no solo, o qual contribui para um melhor controle da abertura e fechamento dos estômatos das plantas, bem como na ativação de várias enzimas e síntese de proteínas (MARSCHNER, 1997).

Comparando os híbridos, observaram-se, em termos médios, maiores teores de $\mathrm{N}, \mathrm{P}, \mathrm{K}$, Ca e Mg alocados na parte aérea e no tronco dos híbridos provenientes de Eucalyptus urophylla quando em polinização com híbridos naturais (Tabela 3). Dessa forma, esses híbridos além de mais produtivos foram os que acumularam maiores quantidades de nutrientes na parte aérea, indicando maior eficiência na absorção dos nutrientes. Segundo Saric (1983), a eficiência na absorção de nutrientes do solo pode ser inferida pelo conteúdo total de nutriente na biomassa, quando as plantas são submetidas a condições de baixa 
disponibilidade de nutrientes, altos conteúdos caracterizam alta eficiência de absorção, podendo resultar em genótipos altamente eficientes em absorver e utilizar os nutrientes.

A distribuição relativa dos nutrientes no tronco, em relação à biomassa aérea total, mostrou maiores teores de P (62,3\%), K (73,1\%), Ca (77,3\%) e Mg (63,6\%) no tronco dos híbridos de Eucalyptus urophylla provenientes de polinização controlada com descendentes do cruzamento de Eucalyptus camaldulensis com Eucalyptus grandis e de N (53,8\%) nos híbridos de Eucalyptus urophylla provenientes de polinização controlada com híbridos naturais, em termos médios (Tabela 3). Desta forma, verifica-se que os híbridos de Eucalyptus urophylla provenientes de polinização controlada com descendentes do cruzamento de Eucalyptus camaldulensis com Eucalyptus grandis, além de absorverem menores teores de nutrientes, acumulam maior proporção relativa dos nutrientes no tronco do que na copa, e provavelmente, isto, possa ter contribuído para a menor produtividade apresentada por esses híbridos (Tabela 2). Zaia e Gama-Rodrigues (2004) citam que o maior acúmulo de nutrientes no tronco se deve ao fato desse componente alocar maior porção da biomassa da parte aérea, em torno de 87,9\%, em média. Porém, considerando que o tronco geralmente é a parte da árvore explorada comercialmente, poderá haver maior exportação de nutrientes nesse componente nos híbridos de Eucalyptus urophylla com descendentes do cruzamento de Eucalyptus camaldulensis com Eucalyptus grandis, o que poderá comprometer a capacidade produtiva do sítio.

Em geral, há boa relação entre a taxa de crescimento e a de acúmulo de nutrientes em eucalipto (GONÇALVES et al., 1997), fato que foi constatado para Eucalyptus grandis e Eucalyptus saligna em São Paulo (SANTANA et al., 1999). Dessa forma, comparando-se os teores de nutrientes alocados na parte aérea do clone I224 (mais produtivo) em relação ao clone HCT-037 (menos produtivo), constatou-se acúmulo de 206,3 $\mathrm{kg} \mathrm{ha}^{-1} \mathrm{de}$ $\mathrm{N}, 11,3 \mathrm{~kg} \mathrm{ha}^{-1}$ de P, 63,6 $\mathrm{kg} \mathrm{ha}^{-1}$ de K, 216,0 $\mathrm{kg} \mathrm{ha}^{-1}$ de Ca e 37,1 $\mathrm{kg} \mathrm{ha}^{-1}$ de Mg. Porém, quando o componente considerado é o tronco, verificou-se que os teores de nutrientes acumulados foram de $130,5 \mathrm{~kg} \mathrm{ha}^{-1} \mathrm{de} \mathrm{N}, 6,9 \mathrm{~kg}$ $\mathrm{ha}^{-1}$ de P, 49,3 $\mathrm{kg} \mathrm{ha}^{-1}$ de K, 187,2 $\mathrm{kg} \mathrm{ha}^{-1}$ de Ca e 15,3 $\mathrm{kg} \mathrm{ha}^{-1}$ de Mg em favor do clone mais produtivo (Tabela 3). Assim, se houver a manutenção da copa do eucalipto no campo após a sua colheita, retornarão para o solo cerca de 75,8 $\mathrm{kg} \mathrm{ha}^{-1}$ de N, 4,4 $\mathrm{kg} \mathrm{ha}^{-1}$ de P, 14,3 $\mathrm{kg} \mathrm{ha}^{-1}$ de K, 28,8 $\mathrm{kg} \mathrm{ha}^{-1}$ de Ca e 21,8 $\mathrm{kg} \mathrm{ha}^{-1} \mathrm{de} \mathrm{Mg}$, valores que poderão ser maiores caso a colheita do eucalipto se restrinja apenas ao lenho (madeira), o que poderá contribuir para a reposição de nutrientes para o solo, e ainda, para a sua conservação, o que é de fundamental importância quando se consideram solos com baixos teores de nutrientes em formas disponíveis e totais, conforme verificado na região de Itamarandiba (MG) (BARROS et al., 1990; MOLICA, 1992; GALO, 1993). Além disso, Santana et al. (1999) comentam que a exportação de elevadas quantidades de nutrientes pela colheita florestal ao longo das rotações poderá levar à redução da capacidade produtiva do sítio.

Quanto à eficiência de utilização dos nutrientes (EUN), medida pelo coeficiente de utilização biológico (CUB), os híbridos mostraram diferenças significativas quanto à produção de biomassa de tronco por quilograma dos nutrientes utilizados e, em termos médios, observou-se maior EUN para os híbridos provenientes de Eucalyptus urophylla quando em polinização natural, em relação aos demais híbridos (Tabela 4). Caldeira et al. (2002) comentam que uma espécie eficiente do ponto de vista nutricional é aquela capaz de sintetizar o máximo de biomassa por nutriente absorvido. Dessa forma, quanto menor for a quantidade de nutriente acumulado no componente arbóreo, maiores serão os valores dos coeficientes de utilização biológica (MOURA et al., 2006) e, conseqüentemente, menor poderá ser a exportação de nutrientes (ZAIA e GAMA-RODRIGUES, 2004). Contudo, Caradus (1992) salienta que o baixo teor de nutriente nos tecidos vegetais ou uma elevada eficiência de utilização de nutrientes podem estar associados a ineficiências, como a baixa translocação das raízes para a parte aérea, ou refletirem uma deficiência nutricional.

A EUN decresceu na seguinte ordem: $\mathrm{P}>\mathrm{Mg}>\mathrm{K}>\mathrm{N}>\mathrm{Ca}$. Fato que também foi registrado por Santana et al. (2000) e Santana et al. (2002). De acordo com esses autores, a variação na EUN pode ser em razão das características intrínsecas do material genético, tais como a não-obtenção do equilíbrio nutricional ótimo ou crítico entre solo, planta e todos os nutrientes, ou seja, pode ter ocorrido limitação de um ou mais nutrientes disponíveis; e as relações hídricas. Além disso, a seleção de material genético, que possua alta eficiência de utilização de todos os nutrientes, parece ser pouco provável, conforme constatado por vários autores (MORAIS et al., 1990; MOLICA, 1992; SANTANA et al., 2002). 
TABELA 4: Coeficiente de utilização biológica (CUB) da biomassa de tronco de híbridos interespecíficos de Eucalyptus spp aos 57 meses de cultivo.

TABLE 4: Coefficient of biological use (CUB) of the log biomass of interspecific hybrids of Eucalyptus spp at 57 months of cultivation.

\begin{tabular}{|c|c|c|c|c|c|c|}
\hline \multirow{2}{*}{ Híbridos } & \multirow{2}{*}{ Clone } & $\mathrm{N}$ & $\mathrm{P}$ & $\mathrm{K}$ & $\mathrm{Ca}$ & $\mathrm{Mg}$ \\
\hline & & \multicolumn{5}{|c|}{ kg de biomassa/kg de nutriente } \\
\hline UR x --- & I-224 & 754,8 E & 12571,5 B & 1306,8 B & 570,0 C & 4219,1 B \\
\hline UR x --- & I-060 & 765,3 E & 12868,8 B & 1205,3 C & 960,7 B & 4279,7 B \\
\hline UR x --- & I-144 & 1087,8 A & 12647,7 B & 1342,4 B & 831,6 B & 3795,7 B \\
\hline UR x --- & I-225 & 889,7 D & $14690,2 \mathrm{~A}$ & 1487,8 A & 880,0 B & $5012,0 \mathrm{~A}$ \\
\hline UR x --- & $\mathrm{I}-044$ & $771,6 \mathrm{E}$ & $12138,8 \mathrm{C}$ & $1158,6 \mathrm{D}$ & $824,8 \mathrm{~B}$ & 4240,8 B \\
\hline Média & & 853,9 & 12983,4 & 1300,2 & 813,4 & 4309,5 \\
\hline UR x GR & HC-249 & $735,1 \mathrm{~F}$ & $12612,8 \mathrm{~B}$ & $1302,3 \mathrm{~B}$ & $851,1 \mathrm{~B}$ & $4844,8 \mathrm{~A}$ \\
\hline UR x GR & HC-232 & $774,2 \mathrm{E}$ & 10218,8 D & $1069,0 \mathrm{E}$ & 665,6 C & $2810,3 \mathrm{D}$ \\
\hline UR x GR & HC-373 & 1024,4 B & 12883,2 B & $1453,0 \mathrm{~A}$ & $1059,0 \mathrm{~A}$ & 3979,4 B \\
\hline UR x GR & HC-257 & $842,5 \mathrm{D}$ & 10490,7 D & $1125,7 \mathrm{D}$ & $652,9 \mathrm{C}$ & 3453,5 C \\
\hline UR x GR & HC-344 & $863,0 \mathrm{D}$ & $8194,1 \mathrm{~F}$ & $1057,2 \mathrm{E}$ & $625,3 \mathrm{C}$ & $2721,2 \mathrm{D}$ \\
\hline UR x GR & HC-289 & $743,9 \mathrm{~F}$ & $9149,1 \mathrm{E}$ & $1055,7 \mathrm{E}$ & $882,0 \mathrm{~B}$ & $2935,8 \mathrm{D}$ \\
\hline Média & & 830,5 & 10591,4 & 1177,1 & 789,3 & 3457,5 \\
\hline UR x (CA x GR) & HCT-041 & $984,9 \mathrm{C}$ & $9972,7 \mathrm{D}$ & $1118,8 \mathrm{D}$ & $604,9 \mathrm{C}$ & $2670,3 \mathrm{D}$ \\
\hline UR $x(C A \times G R)$ & НCT-027 & $792,6 \mathrm{E}$ & 9743,9 D & $1112,2 \mathrm{D}$ & 880,7 B & $3429,8 \mathrm{C}$ \\
\hline UR x (CA x GR) & HCT-037 & $795,5 \mathrm{E}$ & $11523,0 \mathrm{C}$ & $949,2 \mathrm{~F}$ & $674,7 \mathrm{C}$ & $3068,2 \mathrm{D}$ \\
\hline Média & & 857,7 & 10413,2 & 1060,0 & 720,1 & 3056,1 \\
\hline $\mathrm{CV}(\%)$ & & 2,3 & 3,8 & 4,4 & 7,4 & 7,7 \\
\hline
\end{tabular}

Em que: Médias seguidas das mesmas letras, em cada coluna, não diferem entre si, a 5\% de probabilidade pelo teste Scott-Knott.

A baixa eficiência de utilização de Ca pode ser atribuída ao elevado teor desse elemento na casca do eucalipto (SANTANA et al., 2000; SANTANA et al., 2002) e também por este ser pouco móvel no floema (MARSCHNER, 1997), e ainda, a inativação de Ca, por causa da ligação e/ou precipitação na forma de oxalato ou fosfato de cálcio (CALDEIRA et al., 2002).

Em relação ao N, verificou-se que esse elemento, depois do Ca, foi o que apresentou menor eficiência de utilização, no geral, para os híbridos avaliados. Segundo Caldeira et al. (2002), o N possui eficiência de uso relativamente baixa, quando comparado aos outros nutrientes, pelos altos teores nas folhas verdes e pela retranslocação interna, voltando ao solo por meio da queda de serapilheira, sendo assim novamente integrado ao ciclo biogeoquímico.

A maior eficiência de utilização de N foi verificada para o clone I-144, de P e Mg para o clone I-225, o qual juntamente com o clone HC-373 foram mais eficientes na utilização de K. O último clone ainda foi mais eficiente na utilização de Ca (Tabela 4). Dessa forma, verifica-se que o clone mais produtivo (I-224) não apresentou maior eficiência de utilização para todos os nutrientes. Santana et al. (2002) também verificaram que as maiores produtividades obtidas para o Eucalyptus grandis e Eucalyptus saligna não foram acompanhadas de maiores valores de eficiência de utilização dos nutrientes e acrescentaram que tal fato é desejável para a sustentabilidade da produção florestal, uma vez que poderia ocorrer o depauperamento dos solos em termos nutricionais. De acordo com Silveira e Malavolta (2000) cada material genético tem habilidade diferenciada de uso de um ou outro nutriente absorvido. $\mathrm{O}$ que se deve às diferenças genotípicas que podem ocorrer e da interação genótipo-ambiente, e assim alterar a capacidade de absorção, transporte e utilização dos nutrientes pelas plantas (MARSCHNER, 1997).

A EUN para um determinado nutriente pode variar à medida que sua disponibilidade no solo sofra alguma alteração. Em geral, a EUN pelas plantas aumenta com a redução de sua disponibilidade no solo (BARROS et al., 1986). Nesse sentido, Souza (1994) constatou valores de 541 a 937 kg de matéria seca de tronco produzida para cada kg de K absorvido entre 15 clones de Eucalyptus grandis plantados na região de Quartel Geral (MG), sendo, nesse caso, os maiores valores de EUN obtidos quando o K se apresentava em menores concentrações no solo. 
Galo (1993) constatou valor para a eficiência de utilização de K de 1.300 kg de tronco para cada kg de K absorvido para Eucalyptus grandis aos 78 meses de idade, valor, em termos médio, que foi semelhante ao obtido para os híbridos de Eucalyptus urophylla proveniente de polinização com híbridos naturais (Tabela 4), demonstrando maior pobreza de K no solo da região de Itamarandiba (MG).

Barros et al. (1995) adotam como valores críticos no software NUTRICALC, para a produção de biomassa de tronco de eucalipto, os seguintes valores: $\mathrm{P}=12.000, \mathrm{~K}=1.000, \mathrm{Ca}=600 \mathrm{e} \mathrm{Mg}=3.000 \mathrm{~kg}$ de biomassa de tronco $/ \mathrm{kg}$ de nutriente. Com base nesses valores, verificou-se que a eficiência de utilização de $\mathrm{P}, \mathrm{K}$, Ca e Mg, obtidos para a produção de matéria seca de tronco para os clones provenientes de Eucalyptus urophylla advindos de polinização com híbridos naturais, foram ligeiramente superiores aos valores críticos. Enquanto que, para os demais clones apenas $\mathrm{K}$ e Ca, foram superiores aos valores críticos, à exceção do clone HCT-037 (Tabela 4). Contudo, os valores de EUN foram muito semelhantes àqueles considerados como críticos por Barros et al. (1995). Santana et al. (2002) citam que o valor de EUN extremamente alta em relação à EUN crítica indica que o nutriente avaliado pode ter limitado o crescimento na rotação atual e limitará o crescimento da rotação ou ciclo subseqüente, a menos que o nutriente seja suprido via fertilizante em quantidades que atendam à demanda do eucalipto. Além disso, a coleta do eucalipto deve-se limitar apenas ao lenho (madeira) com a manutenção dos demais resíduos no campo para reposição dos nutrientes ao solo, bem como contribuir na sua conservação.

\section{CONCLUSÕES}

Híbridos de Eucalyptus urophylla provenientes de polinização natural ou controlada não apresentam diferença na produção de biomassa de copa quando avaliados numa mesma idade.

A polinização controlada de Eucalyptus urophylla com Eucalyptus grandis e com descendentes do cruzamento de Eucalyptus camaldulensis com Eucalyptus grandis reduz a produção de biomassa de tronco na região do Vale do Jequitinhonha.

Híbridos de Eucalyptus urophylla provenientes de polinização com híbridos naturais são mais produtivos e mais eficientes na absorção e utilização de N, P, K, Ca e Mg para a região de estudo.

Híbridos de Eucalyptus urophylla provenientes de descendentes do cruzamento de Eucalyptus camaldulensis com Eucalyptus grandis são menos produtivos e menos eficientes na absorção e utilização de $\mathrm{N}, \mathrm{P}, \mathrm{K}, \mathrm{Ca}$ e Mg.

\section{REFERÊNCIAS BIBLIOGRÁFICAS}

ANTUNES, F. Z. Caracterização climática do cerrado em Minas Gerais. Informe Agropecuário, Belo Horizonte, v. 6, n. 72, p. 52-63, 1980.

ASSIS, T. F. Melhoramento genético do eucalipto. Informe Agropecuário, Belo Horizonte, v. 18, n. 185, p. 32-51, 1996.

ASSIS, T. F. Production of and use of Eucalyptus hybrids for industrial purposes. In: HYBRID BREEDING AND GENETICS OF FOREST TREES SYMPOSIUM, 2000. Queensland, Australia Proceedings... QFRI/CRC-SPF Symposium, 2000. p. 63-74.

ASSIS, T. F.; BAUER, J. F. S.; TAFAREL, G. Sinterização de híbridos de Eucalyptus por cruzamentos controlados. Ciência Florestal, Santa Maria, v. 3, n. 1, p. 161-170, 1993.

ASSOCIATIONS OF OFFICIAL ANALYTICAL CHEMISTS - A.O.A.C. Official methods of analysis of the Associations of Official Analytical Chemists. 12th ed. Washington, D.C., 1975. 1024 p.

BARROS, N. F., NOVAIS, R. F., NEVES, J. C. L. Fertilização e correção do solo para plantio de eucalipto. In: BARROS, N. F.; NOVAIS, R. F. (eds.). Relação Solo-Eucalipto. Viçosa, MG: Folha de Viçosa, 1990. p. 127-186.

BARROS, N. F. et al. Sistema para cálculo del balance nutricional y recomendácion de fertilizantes para el cultivo de eucalipto. Bosque, Valdivia, v.16, p.129-131, 1995.

BARROS, N.F. et al. Classificação nutricional de sítios florestais: descrição de uma metodologia. Revista Árvore, Viçosa, v. 10, n. 2, p. 112-120, 1986.

BRAGA, J. M.; DEFELIPO, B. B. Determinação espectrofotométrica de fósforo em extratos de solos e plantas. Revista Ceres, Viçosa, v. 6, n. 4, p. 73-85, 1974.

CALDEIRA, M. V. W.; RONDON NETO, R. M.; SCHUMACHER, M. V. Avaliação da eficiência nutricional de três procedências australianas de acácia-negra (Acacia mearnsii De Wild.). Revista Árvore, Viçosa, v. 26, n. 5, p. 615-620, 2002.

CALDEIRA, M. V. W.; RONDON NETO, R. M.; SCHUMACHER, M. V. Eficiência do uso de micronutrientes e 
sódio em três procedências de acácia-negra (Acacia mearnsii De Wild.). Revista Árvore, Viçosa, v. 28, n. 1, p. 39-47, 2004.

CARADUS, J. R. Heritability of, and relationships between phosphorus and nitrogen concentration in shoot, stolon and root of while clover (Trifolium repens L.). Plant Soil, Netherlands, v. 146, p. 209-217, 1992.

EMPRESA BRASILEIRA DE PESQUISA AGROPECUÁRIA - EMBRAPA. Serviço Nacional de Levantamento e Conservação de Solos. Sistema Brasileiro de Classificação de Solos. Rio de Janeiro, 1999. 412 p.

GALO, M. V. Resposta do eucalipto à aplicação de potássio em solo de cerrado. Viçosa: UFV, 1993. 40 p. Dissertação (Mestrado em Solos e Nutrição de Plantas) - Universidade Federal de Viçosa, Viçosa.

GOLFARI, L. Zoneamento ecológico do Estado de Minas Gerais para reflorestamento. Belo Horizonte: CEPFRC, 1975. 65 p. (Série Técnica, 3).

GONÇALVES, J. L. M. et al. Soil and stand management for short-rotation plantations. In: NAMBIAR, E. D. S.; BROWN, A. G. (eds.). Management of soil nutrients and water in tropical plantations forest. Canberra: ACIAR, 1997. p. 379-418. (Monograph, 43).

GRAHAM, R. D. Breeding for nutritional characteristics in cereals. In: TINKER, P. B.; LANCHLI, A., (eds.). Advances in plant nutrition. New York: Praeger Publishers, 1984. p. 57-102.

HANSEN, E. A.; BAKER, J. B. Biomass and nutrient removal in short rotation intensively cultured plantations. In: ANNUAL MEETING AMERICAN POPLARS COUNCIL, 1979, Thompsonville. Abstracts...Thompsonville, 1979. p. 130-151.

LADEIRA, B. C. Crescimento, produção de biomassa e eficiência nutricional de Eucalyptus spp., sob três espaçamentos, em uma seqüência de idades. 1999. 132 p. Dissertação (Mestrado em Ciência Florestal) Universidade Federal de Viçosa, Viçosa.

LIMA, A. M. N. et al. Cinética de absorção e eficiência nutricional de $\mathrm{K}^{+}, \mathrm{Ca}^{2+}$ e $\mathrm{Mg}^{2+}$ em plantas jovens de quatro clones de eucalipto. Revista Brasileira de Ciência do Solo, Viçosa, v. 29, p. 903-909, 2005.

MARSCHNER, H. Mineral nutrition of higher plants. $2^{\text {nd }}$ ed. San Diego: Academic Press, 1997. 889 p.

MASON, P. A.; PELHAM, J. Genetic factors affecting the response of trees to mineral nutrients. In: CANNEL, M. G. R.; LAST, F. T., (eds.) Tree Physiology and Yield Improvement. New York: Academic Press, 1976. p.437-448.:

MOLICA, S. G. Produção de biomassa e eficiência nutricional de híbridos interespecíficos de eucalipto, em duas regiões bioclimáticas de Minas Gerais. 1992. 84 p. Tese (Doutorado em Solos e Nutrição de Plantas) - Universidade Federal de Viçosa, Viçosa.

MORAIS, E. J. et al. Biomassa e eficiência nutricional de espécies de eucalipto em duas regiões bioclimáticas de Minas Gerais. Revista Brasileira de Ciência do Solo, Campinas, v. 14, n. 3, p. 353-362, 1990.

MOURA, O. N. et al. Conteúdo de nutrientes na parte aérea e eficiência nutricional em Mimosa caesalpiniifolia Benth. Revista Brasileira de Ciências Agrárias, Recife, v. 1, n. único, p. 23-29, 2006.

ODA, S; MENCK, A. L. M.; VENCOVSKY, R.. Problemas no melhoramento genético clássico do eucalipto em função da alta intensidade de seleção. IPEF, Piracicaba, v. 41/42, n. 3, p. 8-17, 1989.

PEREIRA, A. R. et al. Produção de biomassa e remoção de nutrientes em povoamentos de Eucalyptus citriodora e Eucalyptus saligna cultivados na região do cerrado de Minas Gerais. Revista Floresta, v. 15, n. 1/2, p. 8-16, 1984.

REIS, M. G. F.; BARROS, N. F. Ciclagem interna de nutrientes em plantios de eucalipto. In: BARROS, N. F.; NOVAIS, R. F. (eds.). Relação solo-eucalipto. Viçosa, MG: Folha de Viçosa, 1990. p. 265-302.

SANTANA, R. C.; BARROS, N. F.; COMERFORD, N. B. Aboveground biomass, nutrient content, and nutrient use efficiency of eucalypt plantations growing in different sites in Brazil. New Zealand Journal of Forest Science, Rotorua, v. 30, n. 1, p. 225-236, 2000.

SANTANA, R. C.; BARROS, N. F.; NEVES, J. C. L. Biomassa e conteúdo de nutrientes de procedências de Eucalyptus grandis e Eucalyptus saligna em alguns sítios florestais do Estado de São Paulo. Scientia Forestalis, Piracicaba, v. 56, p. 155-169, 1999.

SANTANA, R. C.; BARROS, N. F.; NEVES, J. C. L. Eficiência de utilização de nutrientes e sustentabilidade da produção em procedências de Eucalyptus grandis e Eucalyptus saligna em sítios florestais do estado de São Paulo. Revista Árvore, Viçosa, v. 26, n. 4, p. 447-457, 2002.

SARIC, M. R. Theoretical and practical approaches to the genetic especificity of mineral nutrition of plants. Plant Soil, Netherlands, v. 72, n. 2, p. 137-150, 1983.

SCHUMACHER, M. V.; POGGIANI, F. Produção de biomassa e remoção de nutrientes em povoamentos de Eucalyptus camaldulensis Dehnh, Eucalyptus grandis Hill ex Maiden e Eucalyptus torelliana F. Muell, plantados em Anhembi, SP. Revista Ciência Florestal, Santa Maria, v. 3, n. 1, p. 21-34, 1993.

SILVEIRA, R. L. V. A.; MALAVOLTA, E. Nutrição e adubação potássica em Eucalyptus. Potafos - Informações 
Agronômicas, Piracicaba, v. 91, 12 p, 2000.

SOUZA, M. E. Correlação adulto-juvenil para a eficiência nutricional e comportamento de clones de Eucalyptus grandis em dois níveis de fertilidade do solo. 1994. 49 p. Dissertação (Mestrado em Solos e Nutrição de Plantas) Universidade Federal de Viçosa, Viçosa.

TEDESCO, M. J. et al. Análises de solo, plantas e outros materiais. Porto Alegre: UFRGS, Departamento de Solos/Faculdade de Agronomia, 1995. 174p. (Boletim Técnico, 5).

ZAIA, F. C.; GAMA-RODRIGUES, A. C. Ciclagem e balanço de nutrientes em povoamentos de eucalipto na região norte fluminense. Revista Brasileira de Ciência do Solo, Viçosa, v. 28, n. 4, p. 843-852, 2004. 